\title{
A ULTRA-SONOGRAFIA DO PÂNCREAS É EFICAZ EM DIAGNOSTICAR O DIABETE MELITO TIPO 1 E TIPO 2?*
}

\author{
Walkyria de Paula Pimenta', Altamir Santos Teixeira², Flávio Ferrari Aragon ${ }^{3}$, \\ Elda Eveline Baptistelli ${ }^{4}$, Adriana Guanaes Moreira ${ }^{4}$, Ana Valéria Barros de Castro ${ }^{5}$, \\ Carlos Roberto Padovani ${ }^{6}$
}

\begin{abstract}
Resumo Este trabalho foi realizado para verificar se a ultra-sonografia do pâncreas oferece dados auxiliares na classificação de diabéticos adultos dos tipos 1 e 2. 0 tamanho e a ecogenicidade do pâncreas foram determinados pela ultra-sonografia em 81 diabéticos, sendo 20 do tipo 1 e 61 do tipo 2 (53 obesos e oito nãoobesos). Os pacientes tipo 2 obesos diferiram dos demais por apresentarem área total e diâmetro ânteroposterior do corpo do pâncreas significativamente maiores. Quanto à ecogenicidade pancreática, esta estava aumentada com maior freqüência nos diabéticos tipo 2 obesos que nos diabéticos tipo 1. Consideramos, assim, que a ultra-sonografia do pâncreas constitui metodologia auxiliar na classificação de diabéticos entre os tipo 1 e 2, sendo menos eficaz quando os últimos não são obesos.

Unitermos: Ultra-sonografia do pâncreas. Pâncreas no diabetes. Tamanho do pâncreas. Ecogenicidade pancreática.
\end{abstract}

\begin{abstract}
Is pancreas ultrasound effective in diagnosing type 1 and type 2 diabetes mellitus?
The aim of this study was to determine whether ultrasonography of the pancreas can be used as an auxiliary tool in the diagnosis of adult patients with type 1 and type 2 diabetes mellitus. The size and echogenicity of the pancreas were evaluated by ultrasound in 81 diabetic patients: 20 type 1 and 61 type 2 (53 obese and 8 non-obese). Obese type 2 diabetic patients showed different results from patients in the other groups, with significantly larger pancreas area and anteroposterior body diameter. The pancreas was more frequently hyperechogenic in obese type 2 diabetic patients than in type 1 diabetic patients. We concluded that ultrasonography of the pancreas may be used as an auxiliary tool for the diagnosis of type 1 and type 2 diabetic patients, although this method showed to be less efficient in non-obese patients.

Key words: Pancreas ultrasound. Pancreas in diabetes. Pancreas size. Pancreas ecogenicity.
\end{abstract}

\section{INTRODUÇÃO}

A classificação de indivíduos adultos com diabetes melito primário (DM) em seus tipos 1 ou 2 tem sido mais difícil nos últimos anos ${ }^{(\mathbf{1})}$. A dosagem do anticorpo antidescarboxilase do ácido glutâmico tem sido de grande auxílio, tendo permitido a

\footnotetext{
* Trabalho realizado no Hospital das Clínicas da Faculdade de Medicina de Botucatu - Universidade Estadual Paulista Júlio de Mesquita Filho (Unesp), Botucatu, SP.

1. Professora Adjunta do Departamento de Clínica Médica da Faculdade de Medicina de Botucatu - Unesp.

2. Professor Assistente do Departamento de Radiologia da Faculdade de Medicina de Botucatu-Unesp.

3. Professor Assistente Doutor do Departamento de Bioestatística do Instituto de Biociências de Botucatu - Unesp.

4. Acadêmicas de Medicina da Faculdade de Medicina de Botucatu - Unesp.

5. Professora Assistente Doutora do Departamento de Clínica Médica da Faculdade de Medicina de Botucatu Unesp.

6. Professor Titular do Departamento de Bioestatística do Instituto de Biociências de Botucatu-Unesp.

Endereço para correspondência: Profa. Dra. Walkyria de Paula Pimenta. Departamento de Clínica Médica, Faculdade de Medicina de Botucatu-Unesp. Botucatu, SP, 18618-970. E-mail:wpimenta@fmb.unesp.br

Recebido para publicação em 26/2/2001. Aceito, após revisão, em 27/3/2002.
}

Zimmet $^{(2)}$ identificar o diabetes de tipo 1 (DM-1) em $15 \%$ a $20 \%$ de diabéticos adultos e em $50 \%$ de diabéticos previamente classificados como do tipo 2 (DM-2) nãoobesos. Todavia, esta metodologia exige tecnologia mais avançada e maiores recursos, nem sempre disponíveis.

Por outro lado, o DM caracteriza-se por deficiência absoluta ou relativa de insulina, que resulta de mecanismos patogênicos diversos para o DM-1 e o DM- $2^{(\mathbf{1})}$.

No DM-1, um processo auto-imunológico causa destruição quase total das células $\beta$ pancreáticas ${ }^{\text {(3-5); }}$ a velocidade de seu desenvolvimento é variável, sendo mais lenta quando acomete indivíduos mais ve$\operatorname{lhos}^{(\mathbf{1}, 2,5,6)}$. No DM-2 há uma disfunção das células $\beta$ pancreáticas, acompanhada de diversos graus de resistência à insulina, de origem genética ainda pouco esclareci$\mathrm{da}^{(\mathbf{7 , 8})}$. Neste tipo de diabetes os resultados são mais controversos; todavia, estudos cuidadosamente conduzidos verificaram redução de $50 \%$ a $60 \%$ do volume das células $\beta$ relativamente ao grupo controle ${ }^{(\mathbf{4 , 9})}$.
A insulina exerce efeito trófico sobre a porção exócrina do pâncreas; desse modo, após alguns anos de evolução do DM-1, ocorre uma redução do volume pancreático. No DM-2, além de essa redução ser menor, há fatores interferentes como a deposição de substância amilóide, a infiltração gordurosa e a presença de estimulantes das células $\beta$, como a resistência insulínica ${ }^{(7,10)}$.

A avaliação morfológica do pâncreas oferece características distintas para ambos os tipos de diabetes e pode ser realizada de forma não-invasiva, desde o advento da ultra-sonografia (US) e da tomografia computadorizada (TC). A primeira técnica tem vantagens em relação à segunda por ser mais simples, menos custosa e não envolver o uso de contrastes radioativos ${ }^{(\mathbf{1 1})}$.

Assim, baseando-se nas evidências de que os comprometimentos funcional e estrutural do pâncreas resultam de processos etiopatogênicos específicos no DM-1 e DM-2, o objetivo deste trabalho foi o de verificar se a US do pâncreas forneceria dados distintos, auxiliares, no diagnóstico 
destes dois tipos principais do DM, num grupo de pacientes adultos.

\section{CASUÍSTICA E MÉTODOS}

Oitenta e um diabéticos participaram do estudo, todos sem passado de alcoolismo, pancreatite ou dislipidemia grave, após terem sido devidamente esclarecidos e assinado o consentimento por escrito. Os pacientes foram assim classificados: $20 \mathrm{com}$ DM-1 e 61 com DM-2, dos quais 53 eram inicialmente obesos e oito eram não-obe$\operatorname{sos}^{(\mathbf{1})}$. As principais características clínicas e laboratoriais encontram-se na Tabela 1.

Dezoito indivíduos saudáveis - dez obesos (quatro homens/seis mulheres; 36 \pm 14 anos; índice de massa corporal $=34,8$ $\pm 6,7 \mathrm{~kg} / \mathrm{m}^{2}$ ) e oito não-obesos (dois homens/seis mulheres; $37 \pm 17$ anos; índice de massa corporal $\left.=23,8 \pm 1,6 \mathrm{~kg} / \mathrm{m}^{2}\right)-$ constituíram o grupo controle.

Os pacientes e os controles foram submetidos a US do abdome às $8 \mathrm{~h} 00$ da manhã, em posição supina, após 12 horas de jejum, tendo sido observado preparo habitual prévio. Todos os exames de US foram realizados por um dos autores, que desconhecia o grupo a que o paciente pertencia. Utilizou-se aparelho de imagem em tempo real (Toshiba Sonolayer 140 SSH) equipado com transdutor convexo de $3,5 \mathrm{MHz}$.

Opâncreas identificado foi divididoem três regiões: a cabeça e o corpo, como as áreas à direita e à esquerda, respectivamente, da confluência das veias esplênica e mesentérica superior, e a cauda, como a porção que fica anteriormente ao rim esquerdo e estende-se ao interior do hilo esplênico. Como a cauda não foi sempre claramente visualizada e representou somente uma pequena parte da massa pancreática, não foi considerada nas análises. A maior área da secção longitudinal do pâncreas (área) era automaticamente calculada e medidos os diâmetros máximos: a) transversal da cabeça (cabeça), que se considerou da borda externa, contígua à alça duodenal, até a região ístmica; b) ântero-posterior do corpo (corpo), em correspondência ao terço médio da veia esplênica. A ecogenicidade do tecido pancreático foi avaliada por comparação àquela do tecido hepático normal e então classificada em hipo, iso ou hiperecogênica.
Tabela1 Características clínicas e laboratoriais dos grupos estudados.

\begin{tabular}{|l|c|c|c|}
\hline & Diabéticos tipo 1 & $\begin{array}{c}\text { Diabéticos tipo 2 } \\
\text { não-obesos }\end{array}$ & $\begin{array}{c}\text { Diabéticos tipo 2 } \\
\text { obesos }\end{array}$ \\
\hline $\mathrm{N}(\mathrm{M} / \mathrm{F})$ & $20(12 / 8)$ & $8(2 / 6)$ & $53(12 / 41)$ \\
Idade (anos) & $26 \pm 8$ & $54 \pm 9$ & $56 \pm 11$ \\
$\mathrm{IMC}\left(\mathrm{kg} / \mathrm{m}^{2}\right)^{*}$ & $25,1 \pm 4,8$ & $24,0 \pm 1,4$ & $31,4 \pm 4,4$ \\
Glicemia anual (mmol/l) & $10,9 \pm 4,7$ & $8,8 \pm 2,8$ & $10,4 \pm 3,5$ \\
Colesterol total (mmol/l) & $4,55 \pm 1,19$ & $5,17 \pm 1,37$ & $5,95 \pm 1,29$ \\
Triglicérides (mmol/l) & $1,17 \pm 0,71$ & $1,52 \pm 1,14$ & $2,55 \pm 2,04$ \\
Duração da diabetes (anos) & $9 \pm 8$ & $9 \pm 9$ & $10 \pm 6$ \\
Tratamento com insulina (\%) & 100,0 & 50,0 & 41,5 \\
Complicações vasculares (\%) & 50,0 & 75,0 & 75,5 \\
\hline
\end{tabular}

Obs.: Dados expressos como média \pm desvio-padrão. M/F, masculino/feminino; IMC, índice de massa corporal.
A comparação das dimensões e da ecogenicidade do pâncreas entre os três grupos de diabéticos e os dois subgrupos controle foi realizada por análise de covariância (Ancova). Esta análise foi complementada com o teste de Tukey para comparação das médias corrigidas ${ }^{(\mathbf{1 2})}$. A análise de associação foi realizada para o grupo dos pacientes com DM-2 obesos pelos coeficientes de Pearson e Spearman, considerando-se cada sexo individualmente ${ }^{(\mathbf{1 3})}$. Considerou-se como nível de significância o valor de $5 \%(\mathrm{P}<0,05)$.

O projeto foi aprovado pelo Comitê de Ética em Pesquisa de nossa Instituição.

\section{RESULTADOS}

Os pacientes com DM-2 obesos apresentaram área pancreática (média \pm desviopadrão(DP)) significativamente maior que a dos demais diabéticos: $21,38 \pm 7,98 \mathrm{~cm}^{2}$ vs. $13,49 \pm 4,98 \mathrm{~cm}^{2}$ nos pacientes com DM-1 e 15,20 \pm 4,57 $\mathrm{cm}^{2}$ nos com DM-2 não-obesos (P < 0,05) (Figuras 1 e 2). $\mathrm{O}$ mesmo foi observado em relação ao corpo do pâncreas (média \pm DP): 1,53 $\pm 0,43 \mathrm{~cm}$ nos pacientes com DM-2 obesosvs. 1,04 $\pm 0,38 \mathrm{~cm}$ nos com DM-1 e 1,24 $\pm 0,18 \mathrm{~cm}$ nos com DM-2 não-obesos ( $\mathrm{P}<0,05)$. Os três grupos não foram diferentes em relação à medida da cabeça pancreática (média \pm DP): $2,62 \pm 0,63 \mathrm{~cm}, 2,27 \pm 0,57 \mathrm{~cm}$ e 2,38 $\pm 0,50 \mathrm{~cm}$ nos pacientes com DM-2 obesos, DM-1 e DM-2 não-obesos, respectivamente $(\mathrm{P}>0,05)$ (Figura 3$)$.

No grupo controle os indivíduos obesos apresentaram área pancreática (média \pm DP) $-19,09 \pm 6,23 \mathrm{~cm}^{2}$ vs. 14,21 $\pm 4,25$ $\mathrm{cm}^{2}$ - e medida do corpo do pâncreas (média \pm DP) $-1,34 \pm 0,25 \mathrm{~cm}$ vs. 1,10 \pm $0,30 \mathrm{~cm}$ - maiores que os indivíduos nãoobesos, a nível de $0,05<\mathrm{P}<0,10$.

Os resultados da ecogenicidade relativa do pâncreas dos três grupos estudados encontram-se na Tabela 2. O grupo com DM-2 obesos apresentou pâncreas hiperecogênico mais freqüentemente que o com DM-1 ( $\mathrm{P}<0,05)$. O pâncreas dos pacientes com DM-2 obesos mostrou-se principalmente hiperecogênico $(72 \%)$, menos vezes isoecogênico (26\%) e raramente hipoecogênico (2\%); os com DM-1 mostraram frequiência semelhante das três categorias de ecogenicidade pancreática: $35 \%$ hiperecogênico, $45 \%$ isoecogênico e $20 \%$ hipoecogênico; o pâncreas dos com DM-2 não-obesos foi hiper ou isoecogênico.

Os indivíduos controles obesos não diferiram dos não-obesos quanto à ecogeni-
Tabela 2 Freqüência de cada grau de ecogenicidade pancreática nos grupos estudados.

\begin{tabular}{|l|c|c|c|c|}
\hline \multirow{2}{*}{} & \multicolumn{3}{|c|}{ Ecogenicidade pancreática } & \multirow{2}{*}{ Total } \\
\cline { 2 - 4 } & Hipo & Iso & Hiper & \\
\hline Diabéticos tipo 1 & $0,200 \mathrm{a}^{*} \mathrm{~A}^{\S}$ & $0,450 \mathrm{aA}$ & $0,350 \mathrm{aA}$ & 20 \\
Diabéticos tipo 2 não-obesos & $0,000 \mathrm{aA}$ & $0,500 \mathrm{aB}$ & $0,500 \mathrm{abB}$ & 8 \\
Diabéticos tipo 2 obesos & $0,019 \mathrm{aA}$ & $0,264 \mathrm{aB}$ & $0,717 \mathrm{bC}$ & 53 \\
\hline
\end{tabular}

* Letras minúsculas foram usadas para comparar os grupos quanto a cada grau de ecogenicidade pancreática. $\S$ Letras maiúsculas foram usadas para comparar a freqüência de cada grau de ecogenicidade pancreática em cada grupo. Dois resultados com pelo menos uma letra do mesmo tipo não são diferentes ao nível de significância de $5 \%$. 


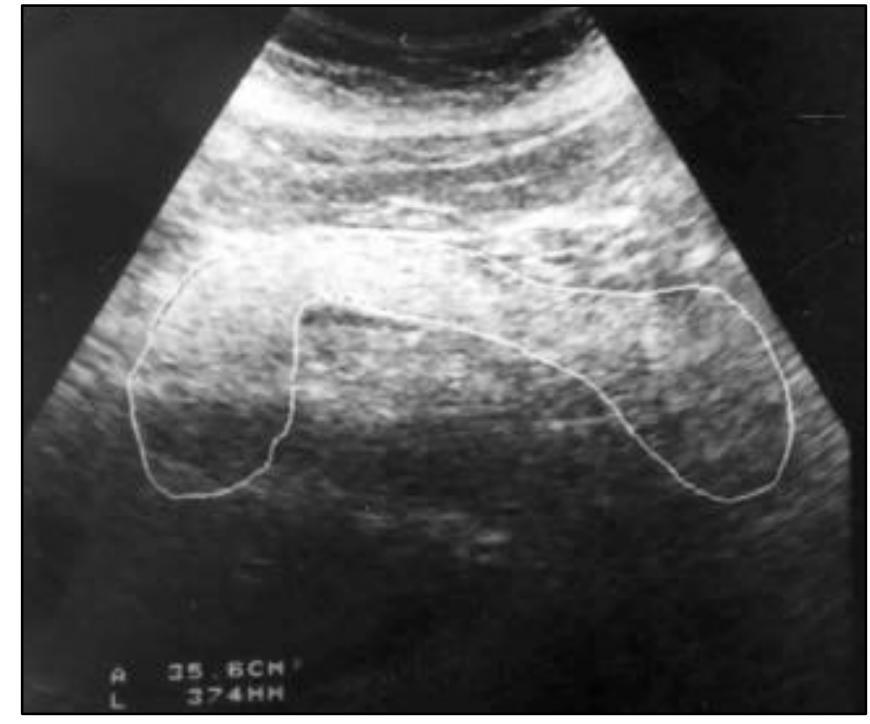

1

Figura 1. Corte transversal. Pâncreas hiperecogênico de um diabético tipo 2, obeso, cujo maior diâmetro longitudinal foi medido e sua área automaticamente calculada em $\mathrm{cm}^{2}$.

Figura 2. Corte transversal. Pâncreas isoecogênico de um diabético tipo 1 , cujo maior diâmetro longitudinal foi medido e sua área automaticamente calculada em $\mathrm{cm}^{2}$.

Figura 3. Corte transversal. Pâncreas isoecogênico de um diabético tipo 2, não-obeso, com medida dos diâmetros máximos transverso e ântero-posterior da cabeça e do corpo do pâncreas, respectivamente.
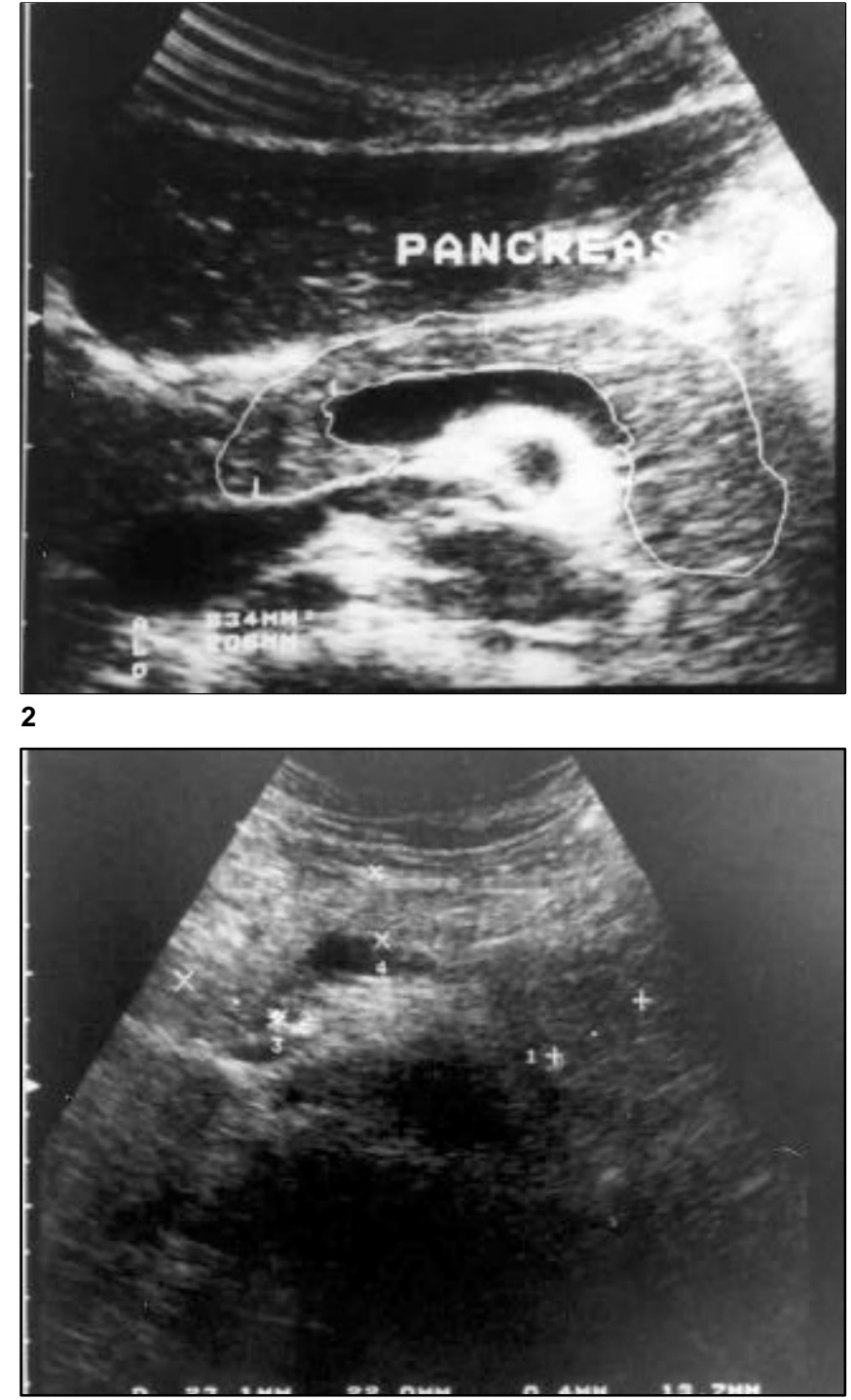

3 cidade do pâncreas, que foi $50 \%$ e $75 \%$ isoecogênico, e $50 \%$ e $25 \%$ hiperecogênico, respectivamente.

O sexo foi a única variável que interferiu na avaliação ultra-sonográfica do pâncreas, tendo sido considerada em todas as análises.

No grupo dos pacientes com DM-2 obesos não houve correlação entre os parâmetros clínico-laboratoriais e as medidas pancreáticas.

\section{DISCUSSÃO}

Com o evoluir dos conhecimentos sobre a etiopatogenia do DM, os limites entre os tipos clássicos 1 e 2 tornaram-se menos precisos, não sendo raro, hoje, na prá- tica clínica, termos dificuldade em classificar um paciente diabético.

São antigos os estudos morfológicos ${ }^{(4,}$ 10) e funcionais ${ }^{(8,14)}$ que demonstraram que o comprometimento fundamental no DM é o das células $\beta$ pancreáticas, com conseqüente menor estimulação da porção exócrina do pâncreas ${ }^{(15)}$. Por processos patogênicos diversos, a lesão das células $\beta$ é mais intensa nos pacientes com DM-1 que nos com DM-2, além de haver, nestes, concomitante reação de infiltração e degeneração do pâncreas.

Dispondo-se de método simples e nãoinvasivo de avaliação morfológica do pâncreas, como a US, consideramos de interesse verificá-la como um meio auxiliar no diagnóstico diferencial entre pacientes com
DM-1 e DM-2 num grupo de diabéticos adultos.

Os diabéticos tipo 2 obesos diferenciaram-se dos com DM-1 e com DM-2 nãoobesos por apresentarem medidas da área total e do corpo do pâncreas significativamente maiores. Tal achado encontra apoio em estudos com diabéticos, seja da população brasileira ${ }^{(16)}$ ou estrangeira ${ }^{(17-20)}$. aumento relativo do tamanho do pâncreas decorreria do fato do comprometimento das células $\beta$ ser principalmente funcional e menos morfológico ${ }^{(21)}$ e do exame de US do pâncreas não diferenciar entre tecido glandular e os depósitos de gordura e de substância amilóide. Neste estudo pudemos verificar efeito expressivo da obesidade em aumentar o tamanho do pâncreas. 
As dimensões pancreáticas em pacientes com DM-2 não-obesos não foram diferentes das em diabéticos tipo 1 . Sabe-se que a deficiência de insulina nestes diabéticos é intermediária entre a apresentada pelos pacientes com DM-1 e a pelos pacientes com DM-2 obesos, o que deveria corresponder a um grau intermediário de redução do tamanho do pâncreas. De fato, em 19 pacientes com DM-2 recebendo insulina, Fonseca et al. ${ }^{(\mathbf{1 8})}$ verificaram tamanho do pâncreas menor que o dos demais diabéticos tipo 2, mas maior que o dos com DM1. Ainda, Migdalis et al. ${ }^{(19)}$ e Alzaid et $a l .{ }^{(17)}$ encontraram, no grupo dos pacientes com DM-2, uma correlação positiva entre as dimensões pancreáticas e a secreção de peptídio C. Nossos resultados dever-seiam, então, ao reduzido número destes diabéticos e à possibilidade de estarem incluídos, neste grupo, alguns que na realidade seriam do tipo 1 com evolução mais lenta.

O tamanho do pâncreas dos diabéticos tipo 1 foi menor que o dos diabéticos tipo 2 , como verificado por outros autores ${ }^{(\mathbf{1 6}-}$ ${ }^{\mathbf{1 8 , 2 0})}$; todavia, esta diferença foi signficativa apenas em relação ao subgrupo obeso, que constitui a maioria dos pacientes com DM-2. Além disso, 50\% de nossos pacientes com DM-1 tinham tempo de doença menor que dez anos e o processo auto-imunológico de destruição do pâncreas tem velocidade de evolução variável, exigindo, muitas vezes, tempo maior que aquele para induzir reduções mais acentuadas do volume glandular.

O grau de ecogenicidade do pâncreas é determinado principalmente pelo depósito de gordura, que o envolve, e do septo, que o atravessa. Dessa forma, como variáveis independentes, a idade e a obesidade, principalmente do tipo abdominal, aumentam seu poder ecogênico ${ }^{(\mathbf{1 6 , 2 2})}$. Nos pacientes com DM-2 a presença de obesidade, de idade mais avançada e de processos degenerativo e infiltrativo do tecido pancreático faz com que o pâncreas seja predominantemente hiperecogênico, como verifica- mos, e também Silva etal. ${ }^{(16)}$. Observamos que os indivíduos obesos apresentaram mais vezes pâncreas hiperecogênico que os indivíduos não-obesos, porém tal diferença não foi significativa, provavelmente por causa do número insuficiente de indivíduos estudados. Já os pacientes com DM-2, na ausência de obesidade e por processos degenerativos do pâncreas, possivelmente diversos dos do grupo anterior, apresentaram pâncreas menos vezes hiperecogênico e mais vezes isoecogênico. A maior freqüência de pâncreas hipoecogênico nos pacientes com DM-1 resultaria de serem geralmente jovens não-obesos e terem atrofia do parênquima glandular. O seu valor dependeria das características do grupo populacional estudado, fundamentalmente, da duração do diabetes ${ }^{(\mathbf{2 0})}$.

Entre os diabéticos tipo 2 obesos não encontramos correlação significativa entre as características clínicas e laboratoriais e as medidas do pâncreas. Tal resultado tem sido variável entre os autores, provavelmente na dependência do grupo populacional considerado.

\section{CONCLUSÕES}

Nossos resultados mostram que o tamanho do pâncreas à US não expressa necessariamente seu estado funcional e constitui metodologia auxiliar no diagnóstico diferencial entre diabéticos de tipo 1 e de tipo 2, embora com menor eficácia quando os últimos não são obesos.

\section{REFERÊNCIAS}

1. Report of the Expert Committee on the Diagnosis and Classification of Diabetes Mellitus. Diabetes Care 1997;20:1183-97.

2. Zimmet PZ. The pathogenesis and prevention of diabetes in adults. Genes, autoimmunity, and demography. Diabetes Care 1995;18:1050-64.

3. Atkinson MA, Maclaren NK. The pathogenesis of insulin-dependent diabetes mellitus. N Engl J Med 1994;331:1428-36.

4. Gepts W. Pathologic anatomy of the pancreas in juvenile diabetes mellitus. Diabetes 1965;14:619_ 33.

5. Saito K, Yaginuma N, Takahashi T. Differential volumetry of A, B and D cells in the pancreatic islets of diabetic and nondiabetic subjects. Tohoku J Exp Med 1979;129:273-83.

6. Maclean N, Ogilvie RF. Quantitative estimation of the pancreatic islet tissue in diabetic subjects. Diabetes 1955;4:367-76.

7. Leahy JL. Natural history of $\beta$-cell dysfunction in NIDDM. Diabetes Care 1990;13:992-1010.

8. Polonsky KS, Sturis J, Bell GI. Non-insulin dependent diabetes mellitus: a genetically programmed failure of the beta cell to compensate for insulin resistance. N Engl J Med 1996;334: 777-83.

9. Klöppel G, Löhr M, Habich K, Oberholzer M, Heitz PU. Islet pathology and the pathogenesis of type 1 and type 2 diabetes mellitus revisited. Surv Synth Pathol Res 1985;4:110-25.

10. Westermark P, Wilander E. The influence of amyloid deposits on the islet volume in maturity onset diabetes mellitus. Diabetologia 1978;15:41721.

11. Guerra MB, Gutiérrez LC, Carrasco RT, Arroyo AP. Tamaño y ecogenicidad del páncreas en adultos chilenos: estudio ecotomográfico em 261 pacientes. Rev Med Chil 1995;123:720-6.

12. Montgomery DC. Design and analysis of experiments. New York: John Wiley, 1991.

13. Norman GR, Streiner DL. Biostatistics - the bare essentials. St. Louis: Mosby-Year Book, 1994.

14. Pfeifer MA, Halter JB, Porte D Jr. Insulin secretion in diabetes mellitus. Am J Med 1981;70:579_ 88.

15. Dandona P, Freedman DB, Foo Y, et al. Exocrine pancreatic function in diabetes mellitus. J Clin Pathol 1984;37:302-6.

16. Silva MER, Vezozzo DP, Ursich MJM, Rocha DM, Cerri GG, Wajchenberg BL. Ultrasonographic abnormalities of the pancreas in IDDM and NIDDM patients. Diabetes Care 1993;16:12967.

17. Alzaid A, Aideyan O, Nawaz S. The size of the pancreas in diabetes mellitus. Diabet Med 1993; 10:759-63.

18. Fonseca V, Berger LA, Beckett AG, Dandona P. Size of pancreas in diabetes mellitus: a study based on ultrasound. Br Med J 1985;291:1240_ 1

19. Migdalis IN, Voudouris G, Kalogeropoulou K, Iliopoulou V, Koutoulidis K, Samartzis M. Size of the pancreas in non-insulin-dependent diabetic patients. J Med 1991;22:179-86.

20. Stella SM, Giunta S, Di Cianni G, et al. Il pancreas nel diabete mellito: aspetti ecografici. Radiol Med 1993;85:70-4.

21. Sempoux C, Guiot Y, Dubois D, Moulin P, Rahier J. Human type 2 diabetes: morphological evidence for abnormal $\beta$-cell function. Diabetes 2001;50 (Suppl 1):S172-7.

22. Worthen NJ, Beabeau D. Normal pancreatic echogenicity: relation to age and body fat. AJR 1982;139:1095-8. 